\title{
Radiological predictors of outcome in basilar artery stroke:
}

\section{THE BASILISK STUDY}

L. Martínez Vicente ${ }^{1}$; S. Trillo 1; C. Idoate; C. Aguirre 1; C. Ramos 1; Javier Villacieros-Álvarez 1;

E. Bárcena 2; J.L. Caniego 2; R. Manzanares 2; A. Barbosa 2; J. Garrido 3; M. Jaquete 4; I. Olazarán 4;

A. Ximenez-Carrillo ${ }^{1}$; J. Vivancos 1.

1 Stroke Unit, Department of Neurology. University Hospital of la Princesa, Spain.

2 Department of Radiology. University Hospital of la Princesa, Spain.

3 Department of Methodology. La Princesa Research Institute.

4 Universidad Autónoma de Madrid. University Hospital of la Princesa, Spain.

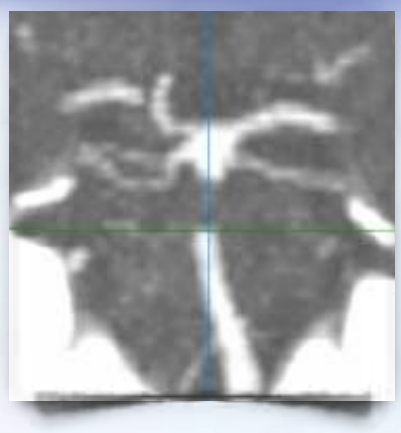

The basilar artery occlusion (BAO) is a medical emergency with a high mortality rate. There are few radiological scores with prognostic capacity in BAO.

BACKGROUND: Our main objective was to study the prognostic capacity of different radiological scale), a new scale for BAO designed in our centre.

We also studied the possible relationship between the predictive capacity of this scales and different clinical and radiological characteristics.

\section{METHODS:}

Retrospective study of patients with BAO treated with endovascular therapy in our hospital, excluding nonrevascularized patients.

Different radiological scales of vertebrobasilar circulation (pcCTA and BATMAN) and the BASILISK scale were applied in CTangiography.

All scales were correlated with outcome, measured with the $\mathrm{mRS}$ at 3 months $(3 \mathrm{~m}-\mathrm{mRS})$.

\section{RESULTS:}

\begin{tabular}{|c|c|c|}
\hline Women & $36 \%(\mathrm{n}=7)$ \\
\hline Mean age (yo) & $60,9 \quad(\mathrm{SD}: 15.3)$ \\
\hline Median NIHSS & $6(3-14)$ \\
\hline Median GSC & $14(6-15)$ \\
\hline Median mRS3m & $3(0-6)$ \\
\hline 3-month death & $21.6 \%(\mathrm{n}=6)$ \\
\hline
\end{tabular}

$$
\mathrm{n}=19
$$

\begin{tabular}{c|c|}
\hline $\begin{array}{c}\text { Mean time from } \\
\text { onset to CT }(\min )\end{array}$ & $\begin{array}{c}246.8(\mathrm{SD}: \\
168.4)\end{array}$ \\
$\begin{array}{c}\text { Mean time from } \\
\text { CT to recanalization }\end{array}$ & $170.3(\mathrm{SD}: 89.8)$ \\
\hline $\begin{array}{c}\text { (min) } \\
\text { Primary thrombectomy }\end{array}$ & $63.2 \% \quad(\mathrm{n}=12)$ \\
\hline Indeterminate etiology & $52.6 \% \quad(\mathrm{n}=10)$ \\
\hline Cardioembolic etiology & $26.3 \%(\mathrm{n}=5)$
\end{tabular}

Scales and its correlation with 3m-mRS

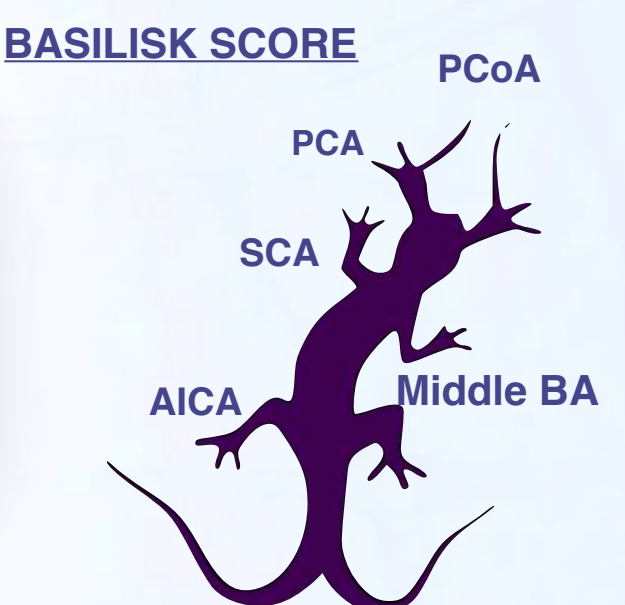

BASILISK

(BASILar Isquemic StroKe scale

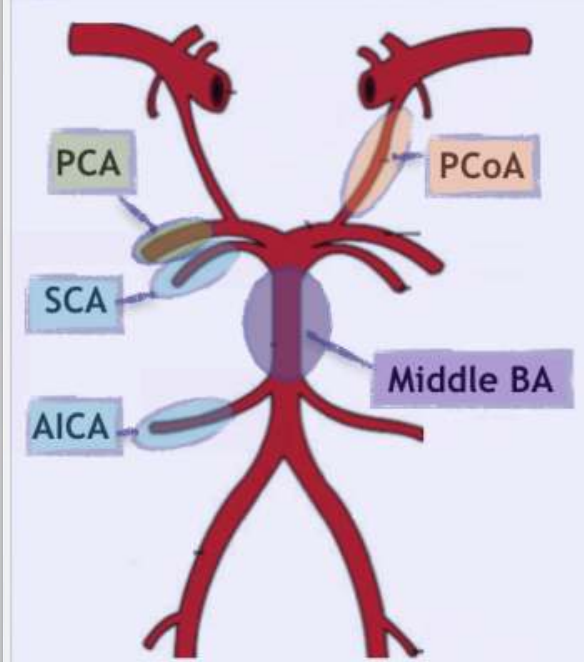

0-10 10: normal circulation

$\mathrm{p}=0,001$ $\mathbf{p}=\mathbf{0 , 0 0 1}$
$($ Rho Spearman $=-0,733)$

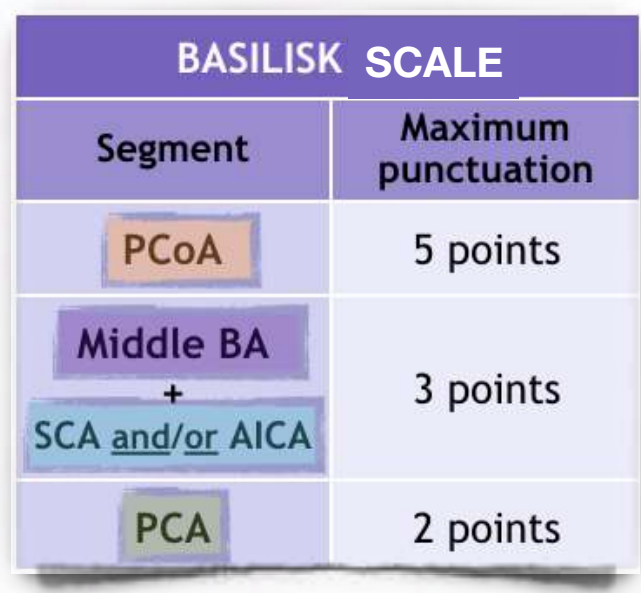

BASILISK and 3m-mRS

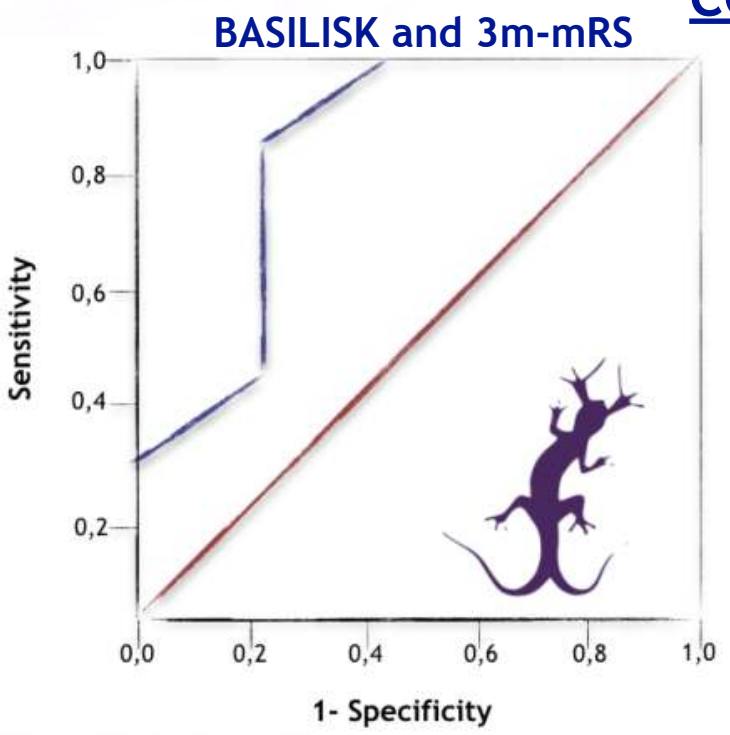

$p=0,03 \quad(A \cup C=0,825)$

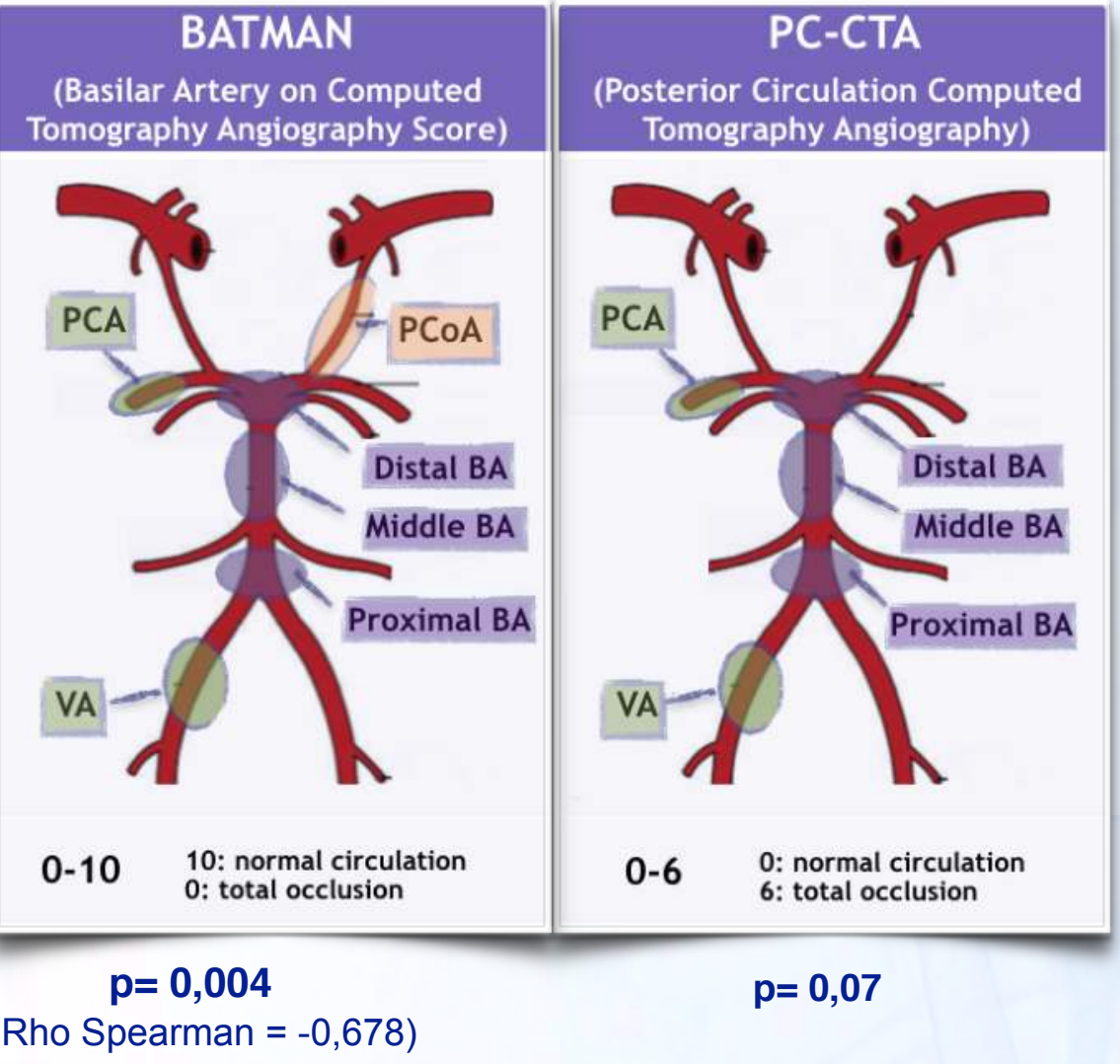

The BASILISK scale is an exponential score with a maximum punctuation of 10 points.

The points are subtracted according to the lack of opacification of different territories of the vertebrobasilar circulation.

We gave a higher value to the absence of posterior communicating artery ( $\mathrm{PCOA})$ and cerebellar arteries located at middle BAO (SCA and AICA) as main collateral pathways.

COR CURVES

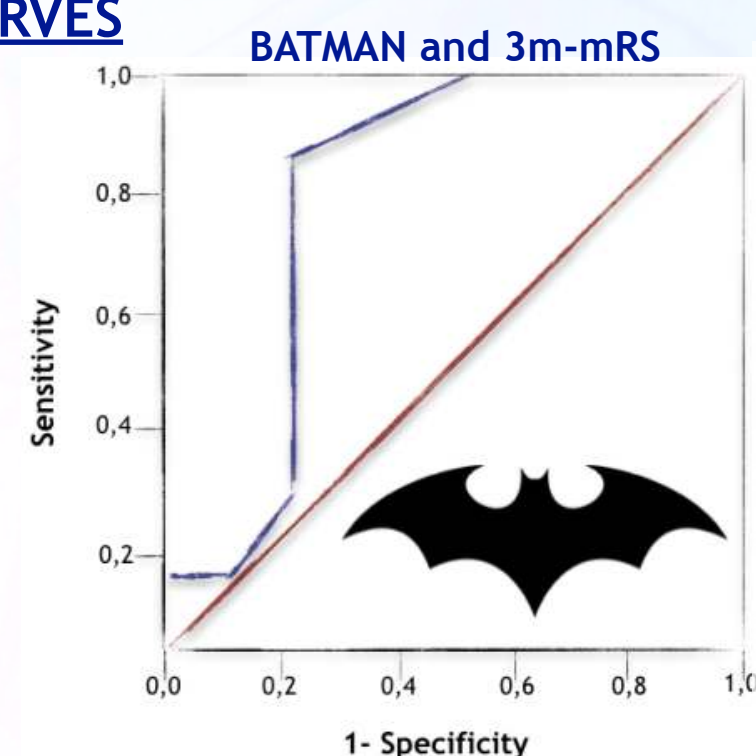

$p=0,05 \quad(A \cup C=0,794)$

\section{CONCLUSIONS:}

Despite the small number of patients in our study, a good prognostic correlation was found with different radiological scales in patients with BAO. There are more ongoing studies to define the usefulness of BASILISK scale in clinical practice.

1. Extent of hypoattenuation on CT angiography source images predicts functional outcome in patients with basilar artery occlusion. Puetz V, et al. Stroke 2008;39(9):2485-90

2. Posterior circulation CT angiography collaterals predict outcome of endovascular acute ischemic stroke therapy for basilar artery occlusion. Goyal N, et al. J Neurolntervent Surg 2015;0:1-

4.3.

3. Collateral flow predicts outcome after basilar artery occlusion: the posterior circulation collateral score. BASICS Registry Investigators. Van der Hoeven, et al; Int $J$ Strok. 2016 0(0) 1-8 .

4. Proposal for a vascular computed tomography-based grading system in posterior circulation stroke: a single-center experience. Da Ros V, et al. J Stroke Cerebrovasc Dis 2016;25(2):368-377

. The Basilar Artery on Computed Tomography Angiography Prognostic Score for Basilar Artery Occlusion. Fana Alemseged, et al. Stroke 2017;48:00-00 